\title{
Intestinal Coinfection with Enterocytozoon bieneusi and Cryptosporidium in a Human Immunodeficiency Virus-Infected Child with Chronic Diarrhea
}

\author{
Rainer Weber, Bärbel Sauer, Ruedi Lüthy, \\ and David Nadal
}

\author{
From the Division of Infectious Diseases. Department of Medicine. \\ University Hospital; and the Division of Immunology and Hematology. \\ University Children's Hospital. Zurich, Switzerland
}

\begin{abstract}
The microsporidian Enterocytozoon bieneusi has been recognized as an important cause of chronic diarrhea in severely immunodeficient adults infected with human immunodeficiency virus (HIV). We report the first case of intestinal $E$. bieneusi infection in a child. The 9-year-old boy with connatal HIV infection presented with failure to thrive, chronic diarrhea, and intermittent abdominal pain. His CD4 lymphocyte count was $0.05 \times 10^{9} / \mathrm{L}$ and dropped to $0.01 \times 10^{9} / \mathrm{L}$. No HIV-associated opportunistic infection other than oral hairy leukoplakia and oral candidiasis had been found before microsporidia were detected. Treatment of microsporidiosis with albendazole was of no benefit. During follow-up, the boy also developed intestinal cryptosporidiosis. Evaluation of chronic diarrhea in severely immunodeficient HIV-infected children should include examination for intestinal microsporidia. We recommend the use of a new coprodiagnostic technique that allows detection of microsporidial spores in stool specimens. Furthermore, consideration of dual or even multiple parasitic infections in the differential diagnosis of chronic diarrhea may have both important clinical and epidemiological implications.
\end{abstract}

The microsporidian protozoan organism Enterocytozoon bieneusi, first reported in 1985 [1, 2], has been recognized as a human immunodeficiency virus (HIV)-associated intestinal opportunistic pathogen [1-9]. Preliminary epidemiological studies have indicated that it may be present in 10\%-30\% of severely immunodeficient HIV-infected adults with chronic diarrhea [3-6, 9].

We report the first case in which an HIV-infected pediatric patient with chronic diarrhea was found to have E. bieneusi spores in stool specimens by light-microscopic and electronmicroscopic examination. During follow-up, the child also developed intestinal cryptosporidiosis.

\section{Case Report}

The patient, a 9-year-old boy, was born in Switzerland to an HIV-seropositive Swiss mother with a history of intravenous drug use. Between the ages of 3 and 8 years, the boy lived with his mother in Ecuador before his return to Switzerland. His mother died of AIDS-related complications in February 1992. She did not have diarrhea. The boy developed normally and was healthy until summer 1991, when he expe-

Received 18 December 1992; revised 10 March 1993.

Grant support: This work was supported in part by a grant from the Swiss National Program for AIDS Research (91-7066).

Reprints or correspondence: Dr. Rainer Weber, Division of Infectious Diseases, Department of Medicine, University Hospital, CH-8091 Zurich, Switzerland.

Clinical Infectious Diseases 1993;17:480-3

(c) 1993 by The University of Chicago. All rights reserved. $1058-4838 / 93 / 1702-0023 \$ 02.00$ rienced intermittent diarrhea, abdominal pain, and mild dry cough without fever.

In September 1991, he was referred to the University Children's Hospital in Zurich because of persistent gastrointestinal symptoms. He had three to six watery stools per day and intermittent nonspecific diffuse abdominal pain. His weight $(19.3 \mathrm{~kg})$ and height $(118 \mathrm{~cm})$ were below the third percentile. Physical examination was normal apart from oral hairy leukoplakia and oral candidiasis. He was found to be positive for antibody to HIV-I, and the results of HIV-I culture also were positive. The hematocrit was 0.31 , the leukocyte count was $4.6 \times 10^{9} / \mathrm{L}$ with a $\mathrm{CD} 4^{+}$count of $0.05 \times 10^{9} / \mathrm{L}$ and a $\mathrm{CD}^{+}$lymphocyte count of $2.03 \times 10^{9} / \mathrm{L}$. Tests of functional immune status disclosed antibody deficiency and cellular unresponsiveness to recall antigens and mitogens. Results of alkaline phosphatase, aspartate aminotransferase, lactate dehydrogenase, creatinine tests and urinalysis were normal. Repeated stool examinations for ova and parasites, including cryptosporidia, and viruses were negative, as were bacterial and mycobacterial stool cultures. The chest roentgenogram and ultrasonographs of the abdomen were normal.

Primary prophylaxis for Pneumocystis carinii pneumonia (trimethoprim-sulfamethoxazole, $36 \mathrm{mg} / \mathrm{kg}$ body weight per day) and monthly substitution with intravenous gammaglobulin $(0.4 \mathrm{~g} / \mathrm{kg}$ body weight) were initiated. Oral candidiasis was treated with fluconazole.

He attended school and was well except for persistent diarrhea, with three to six watery or soft stools per day. During the following 9 months, his $\mathrm{CD}^{+}$lymphocyte count dropped to $0.01 \times 10^{9} / \mathrm{L}$, and both his growth $(+0.7 \mathrm{~cm})$ and weight gain $(+0.5 \mathrm{~kg})$ were poor.

In June 1992 microsporidial spores were found by lightmicroscopic and electron-microscopic examination of stool 


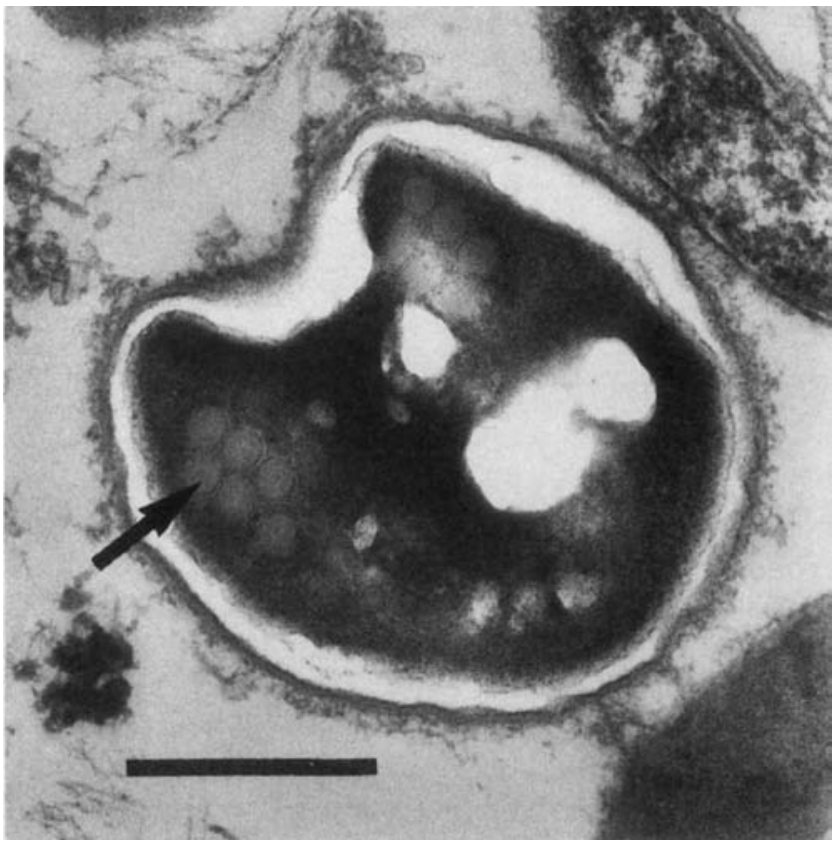

Figure 1. Transmission electron micrograph of an individual microsporidial spore from a stool specimen of an HIV-positive child with chronic diarrhea. The spore is characterized by polar tubes (arrow) and a spore wall consisting of a dense outer spore coat, an electron-lucent endospore layer, and a plasma membrane (bar = $0.5 \mu \mathrm{m})$

specimens with use of a new, recently described coprodiagnostic technique [9] (figure 1). No other intestinal pathogens were detected.

In September 1992, he was hospitalized for 25 days because of severe wasting. He experienced up to 10 watery stools per day. His height was $121 \mathrm{~cm}$, and his weight had dropped to $16.5 \mathrm{~kg}$. His temperature was normal. Electrolyte values were as follows: sodium, $129 \mathrm{mmol} / \mathrm{L}$ (normal, $137-$ 145); potassium, $1.3 \mathrm{mmol} / \mathrm{L}$ (normal, 4.0-5.2); chloride, $95 \mathrm{mmol} / \mathrm{L}$ (normal, 97-107); and calcium, $1.86 \mathrm{mmol} / \mathrm{L}$ (normal, 2.2-2.6). Levels of liver enzymes and creatinine were normal. Stool examination still revealed microsporidial spores. In addition, Cryptosporidium oocysts were found in stool specimens at this time. Because the persistent cough had become more severe and the chest roentgenogram showed small perihilar interstitial infiltrates, bronchoalveolar lavage was performed. No parasites, including cryptosporidia and microsporidia, or mycobacteria were found in the bronchoalveolar fluid, but bacterial cultures were positive for pneumococci. A 10-day course of amoxicillin clavulanate was given. Total parenteral nutrition was started. Albendazole ( $200 \mathrm{mg}$ twice daily) was given for 4 weeks. Monthly intravenous therapy with gammaglobulin was continued. The grandmother caring for the boy refused antiretroviral therapy with zidovudine for the boy.

When the boy left the hospital, he had regained $2.7 \mathrm{~kg}$ and his weight was the same as it had been 1 year earlier (19.2 $\mathrm{kg}$ ). His chest roentgenogram was normal. He still had six watery bowel movements per day and a persistent dry cough. Examination of 16 consecutive stool specimens (before, during, and after the 4-week course of albendazole), including a count of parasites in smears prepared with $20 \mu \mathrm{L}$ of stool suspension, revealed persistent excretion of microsporidial spores (180-240 spores/10 oil-immersion fields at $1000 \times$ magnification) as well as cryptosporidial oocysts (2-7 oocysts/slide).

\section{Detection of Microsporidial Spores in Stool Specimens}

Microsporidial spores were found by light-microscopic examination (1000X magnification, oil immersion) of smears prepared from an unconcentrated suspension of stool in $10 \%$ formalin and stained with a chromotrope-based stain as previously described [9]. The microsporidial spores measured 0.9-1.2 $\times 1.5 \mu \mathrm{m}$ and were ovoid and refractile; the spore wall stained bright pinkish-red. Most spores showed a distinct belt-like stripe stained pinkish-red that girded the spores diagonally or equatorially. The microsporidial species was identified by electron microscopical examination, which showed the typical characteristics of the $E$. bieneusi spore (figure 1) $[3,8,9]$.

\section{Detection of Cryptosporidial Oocysts in Stool Specimens}

Stool samples were processed by an adaption of the formalin-ethyl acetate stool-concentration technique of Ritchie. The concentrates were examined for oocysts by the modified cold Kinyoun acid-fast staining technique and by an indirect immunofluorescence detection procedure according to the instructions of the manufacturer (Crypto-Cel, Cellabs, Brookvale, Australia).

\section{Discussion}

Diarrhea, weight loss, and failure to thrive are common in symptomatic HIV-infected children [10-15]. Nevertheless, epidemiological studies designed to determine the causes of gastrointestinal disease in HIV-infected children as well as the origin and transmission of intestinal pathogens are rare. In an American inner-city hospital, the incidence of diarrhea was 2.6-7 times higher in HIV-seropositive than in HIV-seronegative children [13]. Among 201 symptomatic HIV-infected children in central Africa, $97 \%$ presented with severe weight loss and $62 \%$ presented with diarrhea lasting $>4$ weeks [11]. Whereas the prevalence of Cryptosporidium species and Isospora belli in stool specimens of children in a tropical country was high ( $40 \%$ and $8 \%$, respectively), among HIV-infected pediatric patients with diarrhea [12], a European study found rotavirus in 9 of 20 children, cryptosporidia in 2 , and a bacterial pathogen in 1 [14]. The prevalence 
Table 1. Microsporidiosis in children: summary of case reports.

\begin{tabular}{|c|c|c|c|c|c|}
\hline Microsporidial species & $\begin{array}{l}\text { Child's } \\
\text { age/sex }\end{array}$ & $\begin{array}{c}\text { Clinical } \\
\text { manifestation }\end{array}$ & $\begin{array}{c}\text { Immune } \\
\text { status }\end{array}$ & $\begin{array}{l}\text { Detection } \\
\text { of parasite }\end{array}$ & Year [reference] \\
\hline Encephalitozoon cuniculi & $9 \mathrm{y} / \mathrm{M}$ & Convulsions & Not known & CSF, urine & $1959[18]$ \\
\hline E. cuniculi & $2 \mathrm{y} / \mathrm{M}$ & Convulsions & Low CD4/CD8 ratio & Serum antibody, urine & $1984[21]$ \\
\hline Nosema connori & $4 \mathrm{mo} / \mathrm{M}$ & Disseminated infection & Thymic aplasia & Autopsy & $1973[19]$ \\
\hline Microsporidium ceylonensis & $11 \mathrm{y} / \mathrm{M}$ & Corneal ulcer & Not known & Histological examination & $1973[20]$ \\
\hline Enterocytozoon bieneusi & $9 \mathrm{y} / \mathrm{M}$ & Diarrhea, failure to thrive & $\begin{array}{l}\text { Low CD4 count, HIV } \\
\text { infection }\end{array}$ & Stool specimen & 1993, present case \\
\hline
\end{tabular}

of microsporidiosis in pediatric patients remains to be investigated.

Preliminary epidemiological data have suggested that routine stool examination might detect a potentially treatable infection in $~ 50 \%$ of children with HIV-associated diarrhea [15]. It is not known whether an endoscopic examination would increase the diagnostic yield when a comprehensive examination of stool is negative. Until recently, detection of intestinal E. bieneusi infection has depended on the identification of organisms in small-bowel tissue obtained by endoscopic biopsy [3]. Because of the invasive nature of the endoscopic biopsy, it has not been widely used for pediatric patients with AIDS, thus hampering the ability to detect microsporidia. The most recently described, novel diagnostic technique serves as a practical, noninvasive means of detecting microsporidial spores in stool specimens [9].

Microsporidia are obligate intracellular spore-forming protozoa whose range of hosts includes most invertebrates and all classes of vertebrates [16]. The genera Enterocytozoon, Encephalitozoon, Pleistophora, and Nosema, as well as microsporidian organisms not yet classified, have been associated with human disease primarily in immunocompromised individuals $[16,17]$. More than 150 cases of microsporidiosis in adult patients with AIDS have been described, the majority due to $E$. bieneusi. Among persons not infected with HIV, eight cases of microsporidiosis have been reported, four of them in children [16, 18-21] (table 1).

$E$. bieneusi has been found in small-intestinal enterocytes, duodenal aspirates, and fecal specimens of only those HIVinfected patients who presented with chronic diarrhea $[3,5$, 9]. Epidemiological data have established a causal relation between the presence of the parasite and chronic diarrhea [5, 9]. Furthermore, the parasite has been identified in the biliary tract of some patients with AIDS-related sclerosing cholangitis [22]. Of note, most recently $E$. bieneusi was found in the bronchoalveolar lavage fluid and bronchial epithelium of an HIV-infected patient with chronic cough and small posterobasal infiltrates on chest roentgenogram [23]. The origin of $E$. bieneusi and the mechanisms of its transmission are unknown. Because the organism appears to develop mainly in enterocytes of the small intestine, infection by ingestion of spores is plausible.
The 9-year-old severely immunodeficient boy reported here failed to thrive and had diarrhea that persisted for 16 months of follow-up. No intestinal pathogens, including cryptosporidia, were found in stool specimens until a novel coprodiagnostic technique for detection of $E$. bieneusi became available [9] and intestinal microsporidiosis was diagnosed. Our pediatric patient presented with intermittent nonspecific abdominal pain, a presentation contrasting with that seen in adults with $E$. bieneusi infection, who rarely have abdominal pain. It is not known whether abdominal pain in the boy was related to intestinal microsporidiosis. Intermittent abdominal pain may also be a manifestation of AIDS cholangiopathy, a condition that has been described in adults and in two children [24, 25]. In some patients, AIDS cholangiopathy and AIDS-related sclerosing cholangitis have been related to opportunistic pathogens such as Cryptosporidium species, cytomegalovirus, and-most recentlyE. bieneusi $[22,24,25]$. The boy, however, had no chemical or ultrasonograpic signs of involvement of the biliary tract. After 1 year of follow-up, when gastrointestinal symptoms became most severe, a diagnosis of coinfection with microsporidia and cryptosporidia was made. Although we can not exclude the possibility that cryptosporidiosis was unrecognized before its definitive diagnosis, we feel that this diagnosis was not missed, because stool specimens were repeatedly examined carefully for cryptosporidial oocysts. Nevertheless, epidemiological as well as laboratory data have suggested that coprodiagnostic methods may fail to detect Cryptosporidium oocysts in stool specimens of infected patients $[4,6,26$, 27]. Both the child's increasing gastrointestinal symptoms and nonresponsiveness to albendazole therapy might be explained by the presence of Cryptosporidium infection.

Recent reports have suggested that treatment of microsporidiosis might result in clinical improvement. Preliminary reports of a good response rate among patients treated with metronidazole [5], however, could not be confirmed [28]. Treatment with albendazole led to a significant clinical improvement in some patients, although $E$. bieneusi was still present in biopsy specimens of the small intestine obtained after treatment [28]. In our patient, quantitative excretion of microsporidial spores did not change during a 4-week course of albendazole therapy. 
Evaluation of severely immunodeficient HIV-infected children with chronic diarrhea should include examination for intestinal microsporidia. For such an examination, we recommend the use of the new coprodiagnostic technique [9] that permits detection of microsporidia spores in stool specimens. Furthermore, consideration of dual or even multiple parasitic infections in the differential diagnosis of chronic diarrhea may have both important clinical and epidemiological implications.

\section{Acknowledgments}

The authors are indebted to Ruth Keller, Herbert Kuster, and Professor T. Bächi, for technical help and to Sandra Opravil for editorial assistance.

\section{References}

1. Dobbins WO III, Weinstein WM. Electron microscopy of the intestine and rectum in acquired immunodeficiency syndrome. Gastroenterology $1985 ; 88: 738-49$.

2. Modigliani R, Bories C, le Charpentier Y, et al. Diarrhoea and malabsorption in acquired immune deficiency syndrome: a study of four cases with special emphasis on opportunistic protozoan infestations. Gut 1985;26:179-87.

3. Orenstein JM, Chiang J, Steinberg W, Smith PD, Rotterdam H, Kotler DP. Intestinal microsporidiosis as a cause of diarrhea in human immunodeficiency virus-infected patients: a report of 20 cases. Hum Pathol 1990;21:475-81

4. Kotler DP, Francisco A, Clayton F, Scholes JV, Orenstein JM. Small intestinal injury and parasitic diseases in AIDS. Ann Intern Med 1990;113:444-9.

5. Eeftinck Schattenkerk JKM, van Gool T, van Ketel RJ, et al. Clinical significance of small-intestinal microsporidiosis in HIV-1-infected individuals. Lancet 1991;337:895-8.

6. Greenson JK, Belitsos PC, Yardley JH, Bartlett JG. AIDS enteropathy: occult enteric infections and duodenal mucosal alterations in chronic diarrhea. Ann Intern Med 1991;114:366-72.

7. Shadduck JA. Human microsporidiosis and AIDS. Rev Infect Dis 1989:11:203-7.

8. Bryan RT, Cali A. Owen RL. Spencer HC. Microsporidia: opportunistic pathogens in patients with AIDS. Clin Parasitol 1991;2:1-26.

9. Weber R, Bryan RT, Owen RL. Wilcox CM. Gorelkin L, Visvesvara GS. Improved light-microscopical detection of microsporidia spores in stool and duodenal aspirates. $\mathrm{N}$ Engl J Med 1992;326:161-6.

10. Ammann AJ. The acquired immunodeficiency syndrome in infants and children. Ann Intern Med 1985:103:734-7.

11. Davachi F, Kabongo L. Mayemba N, Ndoko K, Ngoie K, N'Galy B. Clinical findings in 201 symptomatic children with AIDS [abstract ThGO 33]. In: Program and abstracts of the 5th International Confer- ence on AIDS. Montréal: International Development Research Centre, 1989.

12. De Paula MDN, Queiroz W, Janini M, et al. Cryptosporidium sp. and Isospora belli infections in pediatric patients [abstract TBP 196]. In Program and abstracts of the 5th International Conference on AIDS Montréal: International Development Research Centre. 1989.

13. Kotloff KL, Johnson JP, Nair P, Losonsky GA, Young A, Morris JG. Diarrhea and its consequences in HIV-infected children [abstract TBP 263]. In: Program and abstracts of the 5th International Conference on AIDS. Montréal: International Development Research Centre, 1989.

14. Guarino A. Gastrointestinal involvement in children with HIV infection [abstract MB 2258]. In: Program and abstracts of the 7th International Conference on AIDS, Florence, 1991.

15. Santa Lucia MM, Ito HT, Castro MET, De Paula MDN, Queiroz W Diarrheal illness: comparative study between AIDS patients and noninfected children [abstract WB 2028]. In: Program and Abstracts of the 7th International Conference on AIDS, Florence, 1991.

16. Canning EU, Lom J, Dykova I. The microsporidia of vertebrates. New York: Academic Press, 1986.

17. Orenstein JM, Dieterich DT, Kotler DP. Systemic dissemination by a newly recognized intestinal microsporidia species in AIDS. AIDS 1992;6:1143-50.

18. Matsubayashi H, Koike T, Mikata I, Takei H, Hagiwara S. A case of Encephalitozoon-like body infection in man. Arch Pathol 1959;67: $181-7$.

19. Margileth AM, Strano AJ, Chandra R, et al. Disseminated nosematosis in an immunologically compromised infant. Arch Pathol 1973;95 145-50.

20. Ashton N, Wirasinha PA. Encephalitozoonosis (nosematosis) of the cornea. $\mathrm{Br} \mathrm{J}$ Ophthalmol 1973;57:669-74.

21. Bergquist NR, Stintzing G, Smedman L, Waller T, Andersson T. Diagnosis of encephalitozoonosis in man by serological tests. BMJ 1984;288:902

22. Pol S, Romana C, Richard S, et al. Enterocytozoon bieneusi infection in acquired immunodeficiency syndrome-related sclerosing cholangitis. Gastroenterology 1992;102:1778-81.

23. Weber R, Kuster H, Keller R, et al. Pulmonary and intestinal microsporidiosis in a patient with the acquired immunodeficiency syndrome. Am Rev Respir Dis 1992;146:1603-5.

24. Cello JP. Acquired immunodeficiency syndrome cholangiopathy: spectrum of disease. Am J Med 1989;86:539-46.

25. Rusin JA, Sivit CJ. Rakusan TA. Chandra RS. AIDS-related cholangitis in children: sonographic findings. AJR Am J Roentgenol 1992; 159:626-7.

26. Weber R, Bryan RT, Bishop HS, Wahlquist SP, Sullivan JJ, Juranek DD. Threshold of detection of Cryptosporidium oocysts in human stool specimens: evidence for low sensitivity of current diagnostic methods. J Clin Microbiol 1991;29:1323-7.

27. Roberts WG, Green PH, Ma J, Carr M, Ginsberg AM. Prevalence of cryptosporidiosis in patients undergoing endoscopy: evidence for an asymptomatic carrier state. Am J Med 1989;87:537-9.

28. Blanshard C, Ellis DS, Tovey DG, Dowell S, Gazzard BG. Treatment of intestinal microsporidiosis with albendazole in patients with AIDS. AIDS 1992;6:311-3. 